\title{
The Roles of Personality Traits and Negative Life Events on the Episodes of Depressive Symptoms in Nonreferred Adolescents: A 1-Year Follow-Up Study
}

\author{
Hao-Jan Yang, Ph.D. ${ }^{\text {a }}$ Yu-Ju Chiu, M.S. ${ }^{\text {, }}$, Wei-Tsuen Soong, M.D. ${ }^{\text {c,d }}$, and \\ Wei J. Chen, M.D., Sc.D. ${ }^{\text {d,e,* }}$ \\ ${ }^{a}$ Department of Public Health, Chung Shan Medical University, Taichung, Taiwan \\ ${ }^{b}$ Department of Psychology, National Taiwan University, Taipei, Taiwan \\ ${ }^{c}$ Department of Psychiatry, St. Joseph's Hospital, Yunlin, Taiwan \\ ${ }^{d}$ Department of Psychiatry, National Taiwan University, Taipei, Taiwan \\ ${ }^{e}$ Institute of Epidemiology, National Taiwan University, Taipei, Taiwan \\ Manuscript received May 13, 2007; manuscript accepted September 4, 2007
}

\begin{abstract}
Purpose: To evaluate the influences of personality traits and nonsevere life events on depressive episodes in a 1-year follow-up in nonreferred adolescents.

Methods: Participants were 2,773 students aged 12 to 16 years randomly selected from six middle schools in Taiwan who were assessed both at baseline for personality traits and depressive symptoms and at 1 year later for negative life events and depressive symptoms. Two, single, or no episodes of depression were based on whether the scores of the Center for Epidemiologic Studies Depression crossed over the cutoff of 28 at two time points. Multinomial logistic regression analyses were used to correlate personality or negative life events, which were trichotomized by means of tertiles or number of events, with the episodes of depressive symptoms.

Results: After controlling for possible confounders, only elevated neuroticism was associated with a single episode of depressive symptoms, whereas a medium level or above of neuroticism, extraversion, or negative life events all exhibited association with the occurrence of two episodes. No interactions were found between all personality traits or between personality and negative life events.

Conclusions: The relations of personality traits or nonsevere life events to depressive symptoms depend on whether the episode is an initial one. A high level in neuroticism is a critical vulnerability factor for the initiation of depressive symptoms in adolescents. Once the threshold has been surpassed, the influences of various personality traits and nonsevere life events on the subsequent depressive symptoms become cumulative and exhibit a dose-response relationship. (C) 2008 Society for Adolescent Medicine. All rights reserved.
\end{abstract}

Keywords:

Personality trait; Life event; Depressive symptom; Adolescents

Personality traits and life events are both potent risk factors for depression. Among personality traits, high levels of neuroticism [1] or low levels of extraversion [2] have

*Address correspondence to: Wei J. Chen, M.D., Sc.D., Institute of Epidemiology, College of Public Health, National Taiwan University, 17 Xuzhou Road, Taipei 100, Taiwan.

E-mail address: wjchen@ntu.edu.tw been associated with depression. Although elevated scores in neuroticism or lowered ones in extraversion might merely reflect depression's severity [3], many prospective studies further found that these personality traits predicted the onset of depressive symptoms [4] and depressive disorders [5]. Personality may also play a role on the recurrence or relapse of depression. The relapse of depression in depressed adult patients was found to be associated with elevated scores in 
neuroticism, but not with lowered scores in extraversion [6], and the neuroticism scores of remitted adults remained higher than those of never-depressed individuals [7]. The differential associations of these two personality traits with depression or its recurrence were found in relatives of depressed adult patients as well [8]. Another line of evidence deriving from twin studies suggested that neuroticism shared substantial genetic contributions with both first onset and recurrence of depression, whereas extraversion did not $[2,9,10]$. Yet, little is known of whether personality traits are associated with the recurrence of depression in adolescents.

Negative or stressful life events have also been associated with an increased risk of both the onset [11] and recurrence [12] of depression. The association of negative life events with the onset of major depression was postulated to be most prominent for the first episode, whereas the association became progressively weaker as the number of previous depressive episodes increased [13]. This kindling model has been supported empirically in both adults [14] and adolescents [15], with a further finding that three or more severe life events representing a critical threshold for the first episode of depression. A meta-analysis revealed that less stressful life events in terms of total number of life events or daily hassles were strongly associated with depressive symptoms in later life [16]. Hence, the effect of stressful life events on depression may depend on the severity of the stress as well as whether the depressive episode is recurrent or not. It remains unclear whether nonsevere events could lead to the sensitization or autonomy on subsequent depression in adolescence [17].

Several clues further imply that the personality traits and life events may not merely be two independent risk factors for depression. A prospective study in women found that the impact of neuroticism on the onset of depression was greater at high than at low levels of adversity [5]. One possibility is that there is common genetic vulnerability to both recurrent depression and accompanied psychosocial risk factors such as neuroticism, stressful life events, or social support [10]. Another possibility is that episodes of depression might leave scars that alter personality in ways that lowered the threshold of life stress required to precipitate depression recurrence [18,19]. In addition, individuals with elevated neuroticism usually cope less efficiently with stress $[20,21]$. Thus, personality traits and life events tend to be intertwined $[5,18]$ and have joint influences on the course of depression [22]. However, whether nonsevere life events in adolescence also have such joint influences with personality traits on depression has been rarely explored.

Adolescence is a period when depressive symptoms start to occur with increased frequencies [23,24], and depressive symptoms emerging in this period usually persist into later life [25] and more likely stay on the course [26]. The aims of this study were thus set to examine whether personality traits or negative life events exerted independent influences on the number of depressive episodes among a cohort of nonreferred adolescents in Taiwan. Possible roles of personality traits and life events on the occurrence or recurrence of depressive symptoms were evaluated by exploring two competing hypotheses. First, the common vulnerability hypothesis assumes that those at risk for depression may also be at risk for personality or stressful life events. Therefore, the influences of personality traits and life events on depressive symptoms will be similar regardless of single or repeated episodes. Second, according to the scarring hypothesis, some factors specifically trigger the initial episode of depressive symptoms and then change the level of other psychosocial factors, which subsequently precipitate repeated episodes of depressive symptoms.

\section{Methods}

\section{Participants}

Participants were recruited from a longitudinal study dated from December 1998 to January 2000 aiming to evaluate the mental health status of school-attending adolescents aged 12 to 16 years in Taiwan. At Wave 1, all the public middle schools in Taipei City were classified into three groups according to the educational levels of residents in the districts where the schools were located (high, medium, and low). One school in each group was selected, and then seven to nine classes were randomly selected from each of the seventh and eighth grades from each school. In addition, one school in Taipei County and one in Tainan City were also selected, in which about half of the classes were randomly selected from each of the seventh and eighth grades. All students of the selected classes not absent on the day of test were invited to participate in this study. To evaluate the reliability and validity of the screening questionnaires with a two-phase design, one more school in Taipei City was selected with all of its students invited to participate in the study. Thus, this school contributed the only ninth graders in the study. A total of 5,992 out of 6,056 eligible students from six schools were selected to participate. The study was approved by the institutional review board of the College of Medicine, National Taiwan University.

The participation in the survey was adopted as a mental health promotion activity by the schools, and hence parental permission was not obtained. All students of selected classes were informed of the aims and requirements of the study at the beginning of the procedure. After informed written consent was obtained from the students, they were asked to complete one depression scale along with a series of demographic and psychosocial measures, including one personality questionnaire. Students with more than two missing items on depression scale or with more than eight missing items on personality questionnaire $(\mathrm{N}=26)$ were excluded from the subsequent analyses. Therefore, there were 5,966 students included at baseline, with males being $50.9 \%$ of the sample and seventh, eighth, and ninth graders 
being $43.5 \%, 40.9 \%$, and $15.5 \%$, respectively. There was no gender difference $\left(\chi^{2}=0.56, \mathrm{df}=1, p=.45\right)$ between our sample and all the middle school students in Taiwan in 1998, according to the enrolled data at the Ministry of Education. However, our subjects were younger than their counterparts of the nation in $1998(p<.0001)$.

One year later at Wave 2, all students in the selected classes at baseline were followed-up except for those in the school from Taipei County, which could not collaborate with test procedures during the follow-up period. In addition, students who had graduated from school at Wave 2 were not followed up. In sum, 3,138 students in the remaining five schools were invited to complete the depression scale again. Life events that occurred in the preceding 12 months were also assessed at this time point. Similar to the approach adopted for the Wave 1, students with more than two missing items on depression scale $(\mathrm{N}=16)$ or life events scale $(\mathrm{N}=3)$ were excluded from the subsequent analyses. Additionally, students who were transferred from other classes at the time of second wave $(\mathrm{N}=358)$ were excluded from the analyses because of their lack of Wave 1 data. For those eligible students, that is, 2,780 as a result of 3,138 minus 358 , only seven were missed at Wave 2 . Because this study focused on evaluating the effects of personality and life events on the change of depressive symptoms across time, students $(\mathrm{N}=2,773)$ with data on depressive symptoms at both Wave 1 and Wave 2, personality, and life events were included in the analyses.

\section{Measures}

Depression. The Center for Epidemiologic Studies Depression (CES-D) Scale [27] was used to evaluate students' depressive symptoms in the past week by summing up the score of its 20 items. The Chinese version of the CES-D has been demonstrated to have satisfactory reliability and validity in Taiwanese adolescents [24]. In the present study, the internal consistency was also found to be good with a Cronbach's alpha of 0.89 .

To delineate the course of depressive symptoms, two strategies were adopted. First, a suitable cutoff point was chosen to define a depressive episode at each time point. Our recent finding suggested that the CES-D score of 28 was an optimal cutoff point for discriminating depressed and nondepressed adolescents in Taiwan [24]. Second, a change in depression status between the two time points was defined by two criteria of different stringency. For the more stringent criterion, a difference of at least five points in the CES-D score was required, which fitted in with the definition of a reliable change [28] and was commonly used in previous studies [29]. For the less stringent criterion, any change in the CES-D score that led to crossover of the cutoff point was treated as a change in depression status. Four types of change in depression during the interval could be classified: depressed at both times (both Wave 1 and
Wave 2 CES-D scores $>28$ ), new episode (Wave 2 CES-D score becoming greater than 28 by an increase from Wave 1 CES-D score of $\leq 28$ ), remission (Wave 2 CES-D score becoming 28 or less by a decrease from Wave 1 CES-D score of $>28$ ), and stay nondepressed (both Wave 1 and Wave 2 CES-D scores $\leq 28$ ).

Another approach in examining the relation of possible risk factors with depression was to sum the number of depressive episodes obtained from the data for each individual, that is, dividing the experience of depression during the follow-up into "two episodes," "single episode," and "no episode."

Personality traits. The Juvenile Eysenck Personality Questionnaire (JEPQ) [30] was used for measuring personality at baseline. It contains 81 yes-no items that constitute three dimensions in terms of neuroticism (20 items), extraversion (24 items), and psychoticism (17 items). The Chinese version of the JEPQ was shown to have good psychometric properties in Taiwanese adolescents [31]. Internal consistencies of neuroticism, extraversion, and psychoticism subscales in the current study were also satisfactory with a Cronbach's alpha of $0.85,0.77$, and 0.70 , respectively. To examine the effects of different levels of personality traits on the number of depressive episode, we divided each dimension into three levels in terms of high, medium, and low, respectively, by means of its tertiles.

Life events. Participants' life events in the past year were assessed at Wave 2 using the Life Event Checklist (LEC), which was specifically designed for older children and adolescents [32]. For each of the 46 events endorsed, the respondent was further inquired about the type (good or bad) and the magnitude of impact (four-point scale, ranging from no, some, moderate, to great effect) of the event. In this study, only bad events with impact were counted. Numbers of events were also divided into three levels: one or less, two or three, and four or more. The Chinese version of the LEC was translated by one senior epidemiologist (W.J.C) after obtaining the permission of Dr. J.H. Johnson. The 28th item "getting your own car" was modified to "getting your own computer" because citizens under the age of 18 in Taiwan are not allowed to drive cars. In this study, the internal consistencies of the LEC were fair to good, ranging from 0.68 for the number of events to 0.70 for the impact of events. The 2-week test-retest reliability of the LEC assessed in a randomly selected subsample $(\mathrm{N}=126)$ was also found to be good, with the intraclass correlation coefficient being 0.86 for the number of negative events.

\section{Data analysis}

Individuals with missing items more than $10 \%$ on the CES-D or the personality subscales were excluded from the analysis. For those who had $10 \%$ or less missing items on the CES-D or JEPQ subscales, we used multiple imputation 
procedure with 10 imputations via the method of Markov Chain Monte Carlo. For the LEC, missing items were treated as skipping unless all the 46 events were missing $(\mathrm{N}=3)$, which were deleted from the analyses.

Multinomial logistic regression analyses were employed to predict the occurrence (single or two episodes) of depressive symptoms by means of personality or negative life events. Initially, the effect of these independent variables was assessed separately, with grade and sex being controlled for. The Jonckheere-Terpstra test for ordered differences among classes on the independent variables was conducted as well. Then those independent variables that exhibited significant association along with their interaction terms were included in a multivariable logistic regression model. Multivariable linear regression models were also performed to examine whether personality traits assessed at baseline were associated with subsequent negative life events at follow-up after controlling for sex and grade of the students. A significant level of 0.05 was set for the Jonckheere-Terpstra test (one-sided) and all the other tests (twosided). All the analyses were conducted using the SAS software version 9.1.

\section{Results}

Participants in this study did not differ from those who were not followed up on gender $\left(\chi^{2}=1.78, p=.18\right)$, extraversion $(t=-0.55, p=.58)$, and psychoticism $(t=$ $0.89, p=0.37)$, but exhibited lower scores in the CES-D ( $t$ $=-5.31, p<.0001)$ and neuroticism $(t=-2.33, p=.02)$ than the dropouts.

The average CES-D scores for the participants at Wave 1 and Wave 2 were $14.3(\mathrm{SD}=9.2)$ and $14.4(\mathrm{SD}=9.4)$, respectively. The proportion of students who were categorized as depressed at Wave $1(7.7 \%)$ was slightly lower than that at Wave 2 (8.8\%; Table 1). The percentages for the four types of course in depression during the interval are also displayed in Table 1. Regardless of the more or less stringent criterion for change in depression was used, the pattern in the course of depression remained similar. The corresponding figures for new episode and remission increased slightly, whereas those for depressed at both times and staying nondepressed decreased slightly. Hence, all the subsequent analyses were based on the five-point change criterion.

The mean scores of the neuroticism, extraversion, and psychoticism for the participants were $9.5(\mathrm{SD}=4.8), 15.3$ $(\mathrm{SD}=4.2)$, and $3.8(\mathrm{SD}=2.4)$, respectively. The average number of negative life events was $1.8(\mathrm{SD}=2.0)$, with the top five most frequently reported events being "making failing grades on report card" $(\mathrm{n}=782)$, "trouble with brother or sister" ( $n=263)$, "increased number of arguments between parents" ( $\mathrm{n}=261)$, "change in parents' financial status" $(\mathrm{n}=256)$, and "increased absence of parent from the home" $(n=249)$. The average impact scores

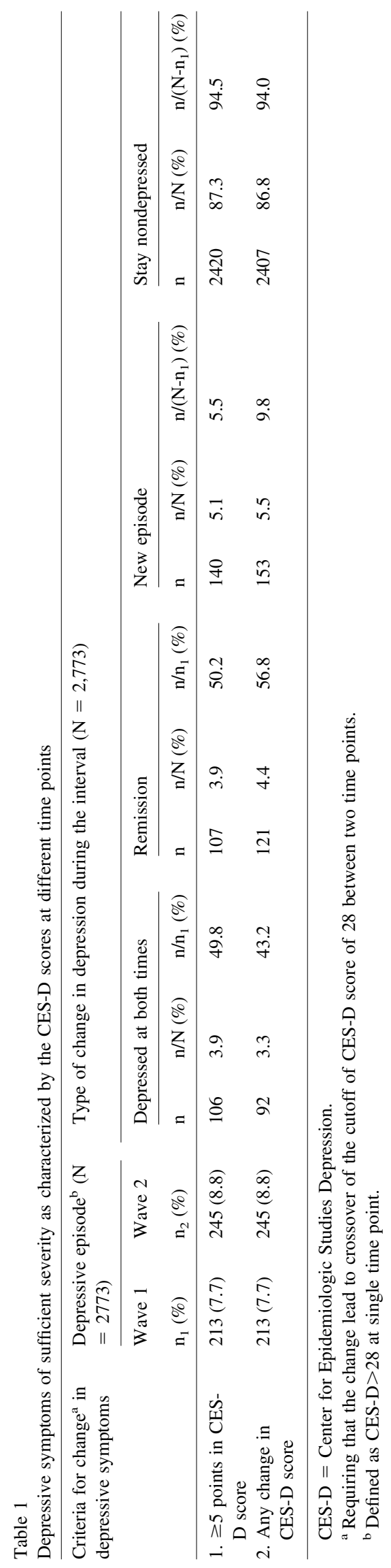


Table 2

Multinomial logistic regression predicting number of episode of depressive symptoms by personality traits and life events separately

\begin{tabular}{|c|c|c|c|c|c|c|c|}
\hline \multirow[t]{2}{*}{ Main independent variable } & \multirow{2}{*}{$\begin{array}{l}\text { No episode } \\
(\mathrm{N}=2,420) \\
\mathrm{n}(\%)\end{array}$} & \multicolumn{2}{|c|}{ Single episode $(\mathrm{N}=247)$} & \multicolumn{2}{|c|}{ Two episodes $(\mathrm{N}=106)$} & \multicolumn{2}{|c|}{$\begin{array}{l}\text { Jonckheere-Terpstra } \\
\text { test }\end{array}$} \\
\hline & & $\mathrm{n}(\%)$ & OR $(95 \% \mathrm{CI})$ & $\mathrm{n}(\%)$ & OR $(95 \% \mathrm{CI})$ & $Z$ & $p$ (one-sided) \\
\hline Neuroticism & & & & & & 19.5 & $<.0001$ \\
\hline Low $(<60 \%$ tile $)$ & $1,590(95.8)$ & $64(3.9)$ & 1.0 & $6(0.4)$ & 1.0 & & \\
\hline Medium (60\%-80\%tile) & $533(86.4)$ & $67(10.9)$ & $1.6(1.0-2.6)$ & $17(2.8)$ & $2.9(1.8-4.6)^{*}$ & & \\
\hline High (>80\%tile) & $297(59.9)$ & $116(23.4)$ & $2.7(1.7-4.2)^{*}$ & $83(16.7)$ & $8.5(5.6-13.0)^{*}$ & & \\
\hline Extraversion & & & & & & -5.4 & $<.0001$ \\
\hline Low (<33\%tile) & $717(82.8)$ & $96(11.1)$ & 1.0 & $53(6.1)$ & 1.0 & & \\
\hline Medium (33\%-66\%tile) & $866(87.7)$ & $87(8.8)$ & $0.8(0.6-1.1)$ & $35(3.5)$ & $0.7(0.6-0.9)^{*}$ & & \\
\hline High $(>66 \%$ tile $)$ & $837(91.1)$ & $64(7.0)$ & $0.7(0.5-1.0)^{*}$ & $18(2.0)$ & $0.5(0.4-0.7)^{*}$ & & \\
\hline Psychoticism & & & & & & 10.4 & $<.0001$ \\
\hline Low $(<33 \%$ tile $)$ & $920(93.4)$ & $47(4.8)$ & 1.0 & $13(1.3)$ & 1.0 & & \\
\hline Medium (33\%-66\%tile) & $844(89.2)$ & $72(7.6)$ & $1.2(0.8-1.7)$ & $30(3.2)$ & $1.6(1.2-2.3)^{*}$ & & \\
\hline High $(>66 \%$ tile $)$ & $656(77.5)$ & $128(15.1)$ & $1.3(0.9-1.8)$ & $63(7.4)$ & $2.9(2.1-3.9)^{*}$ & & \\
\hline Number of negative life events & & & & & & 11.2 & $<.0001$ \\
\hline Low $(0-1)$ & $1,410(92.6)$ & $84(5.5)$ & 1.0 & $28(1.8)$ & 1.0 & & \\
\hline Medium (2-3) & $666(86.5)$ & $69(9.0)$ & $1.2(0.9-1.7)$ & $35(4.6)$ & $1.6(1.3-2.1)^{*}$ & & \\
\hline High $(\geq 4)$ & $344(71.5)$ & $94(19.5)$ & $1.2(0.9-1.6)$ & $43(8.9)$ & $2.5(2.0-3.2)^{*}$ & & \\
\hline
\end{tabular}

All analyses were adjusted for sex and grade; $\mathrm{OR}=$ odds ratio; $\mathrm{CI}=$ confidence interval.

$* p<.05$ for testing the null hypothesis of $\mathrm{OR}=1$.

for the five events were 1.7, 1.2, 1.4, 1.4, and 1.1, respectively, out of a range of 0 to 3 . Meanwhile, regarding severe life events, no girls reported getting pregnant or having abortion, and one boy reported "getting put in jail" (impact $=2.0$ ), one boy reported his girlfriend's having an abortion (impact $=3$ ), and two boys reported their girlfriends' getting pregnant (impact $=3$ ). In general, negative life events reported in this study were mainly nonsevere ones with modest to moderate impacts, whereas those major events with moderate to great impacts were rare.

Using individuals with no episode of depression as the reference group, the odds ratios (ORs) of experiencing single depressive episode or two episodes during the follow-up increased with increment in neuroticism and psychoticism scores, decreased with increment in extraversion scores, and increased with more negative life events, with the trends being significant (all $p \mathrm{~s}<.0001$ by Jonckheere-Terpstra test; Table 2). For the occurrence of single depressive episode, the individual OR reached significance only for the high level in neuroticism $(\mathrm{OR}=2.7)$ as well as the high level in extraversion $(\mathrm{OR}=0.7)$. However, for the occurrence of two depressive episodes, all the personality and life event variables showed significant associations not only for the high level in exposure but also for the medium one. The contrast in the magnitude of OR for the high level exposure versus that for the medium one became more obvious for the occurrence of two depressive episodes than that for single episode. For example, adolescents with medium level in neuroticism had approximately three times the risk $(\mathrm{OR}=2.9)$ of having repeated depressive episodes compared with those with low level in neuroticism, whereas the ratio became 8.5 for students with high level in neuroticism. Similar phenom- ena were observed for extraversion, psychoticism, and number of negative life events, with the contrast in ORs' magnitude becoming less obvious.

The influences of neuroticism, extraversion, psychoticism, and life events were then further evaluated in a multivariable multinomial logistic regression analyses by including all these variables and their pairwise interactions between the most deviant level in each exposure, along with sex and grade. For the occurrence of single depressive episode, the results revealed that only the high level in neuroticism exhibited a significant association $(\mathrm{OR}=3.2$; Table 3). Meanwhile, for the occurrence of two episodes, all the associations but that with the medium level in psychoticism remained, with the magnitude of ORs being similar to those obtained from Table 2. None of the pairwise interac-

Table 3

Multiple multinomial logistic regression of personality traits and negative life events on number of episodes of depressive symptoms

\begin{tabular}{|c|c|c|}
\hline \multirow[t]{2}{*}{ Variables in the model } & Single episode & Two episode \\
\hline & OR $(95 \% \mathrm{CI})$ & OR $(95 \% \mathrm{CI})$ \\
\hline Neuroticism (medium vs. low) & $1.5(0.9-2.5)$ & $2.4(1.5-3.8)^{*}$ \\
\hline (high vs. low) & $3.2(1.8-5.7)^{*}$ & $9.2(5.3-15.8)^{*}$ \\
\hline Extraversion (medium vs. low) & $0.8(0.6-1.1)$ & $0.6(0.5-0.8)^{*}$ \\
\hline (high vs. low) & $0.7(0.3-1.5)$ & $0.5(0.2-0.9)^{*}$ \\
\hline Psychoticism (medium vs. low) & $1.0(0.7-1.5)$ & $1.3(0.9-1.8)$ \\
\hline (high vs. low) & $1.7(1.0-3.1)$ & $2.9(1.7-4.8)^{*}$ \\
\hline $\begin{array}{l}\text { No. of negative life events ( } 2- \\
\quad 3 \text { vs. } 0-1)\end{array}$ & $1.2(0.9-1.6)$ & $1.4(1.1-1.9)^{*}$ \\
\hline ( $\geq 4$ vs. $0-1)$ & $1.1(0.6-1.9)$ & $1.8(1.1-3.2)^{*}$ \\
\hline
\end{tabular}

All analyses were adjusted for sex and grade. $\mathrm{OR}=$ odds ratio.

$* p<.05$ for testing the null hypothesis of $\mathrm{OR}=1$. 
Table 4

Multivariable linear regression models of personality traits at baseline on negative life events at follow-up

\begin{tabular}{|c|c|c|c|c|c|c|c|c|c|}
\hline \multicolumn{4}{|c|}{ Model 1} & \multicolumn{3}{|c|}{ Model 2} & \multicolumn{3}{|c|}{ Model 3} \\
\hline Sex & -0.18 & 0.08 & .0149 & -0.31 & 0.08 & $<.0001$ & -0.41 & 0.08 & $<.0001$ \\
\hline Personality* & 0.11 & 0.01 & $<.0001$ & 0.01 & 0.01 & .1747 & 0.11 & 0.02 & $<.0001$ \\
\hline Model statistics & \multicolumn{3}{|c|}{$\begin{array}{l}F=78.47 ; p<.0001 \\
\text { Adjusted } \mathrm{R}^{2}=.0779\end{array}$} & \multicolumn{3}{|c|}{$\begin{array}{l}F=12.61 ; p<.0001 \\
\text { Adjusted } \mathrm{R}^{2}=.0125\end{array}$} & \multicolumn{3}{|c|}{$\begin{array}{l}F=27.86 ; p<0.0001 \\
\text { Adjusted } \mathrm{R}^{2}=.0284\end{array}$} \\
\hline
\end{tabular}

$\mathrm{SE}=$ standard error.

* Independent variable for personality in models 1, 2, and 3 was neuroticism, extraversion, and psychoticism, respectively.

tion terms were associated with single or repeated episodes of depression and hence were not shown in the table.

We then evaluated whether a depressive episode would predict more negative life events in the future. We found that the number of negative life events reported during the follow-up period was greater for those having a depressive episode at baseline (mean $=3.0, \mathrm{SD}=2.9$ ) than for those without a depressive episode (mean $=1.7, \mathrm{SD}=1.9 ; t=$ $6.36, p<.0001$ ). Whether the personality traits were associated with negative life events was assessed using multivariable linear regression analyses while controlling for sex and grade. We found that the neuroticism (regression coefficient estimate $b=0.11, p<.0001)$ and psychoticism $(b=$ $0.11, p<.0001)$ scores were significantly associated with the number of negative life events, whereas the extraversion scores $(b=0.01, p=.17)$ were not (Table 4$)$.

\section{Discussion}

In this 1-year prospective study, influences of three personality traits and nonsevere negative life events on the occurrence or recurrence of depressive symptoms were examined in a large sample of nonreferred adolescents. Our results revealed that single episode of depressive symptoms was only associated with a high level of neuroticism, whereas repeated episodes were associated with medium or higher levels of neuroticism, extraversion, psychoticism, and negative live events. These are compatible with a psychosocial scar mechanism rather than a common vulnerability in explaining the relations of personality traits and nonsevere negative life events to the course of depressive symptoms.

Although the outcome of this study was categorized by means of a CES-D score of $>28$, the 1-year incidence using a stringent five-point change criteria $(5.5 \%)$ is in line with that of major depressive disorder $(5.7 \%)$ reported previously [33]. The high proportion of depressed students who were still depressed 1 year later $(43.2 \%$ to $49.8 \%)$ in this study appears to be likely the consequences of recurrent symptoms rather than a persistent state. Given the high relapsing rate of depression in adolescents $[34,35]$ and the period of follow-up being 1 year only in this study, we did not distinguish adolescents with single depressive episode into those with remission versus those with new episode in our assessment of their relations to potential risk factors.

For the occurrence of single depressive episode, only those with high level in neuroticism exhibited an increased risk. These results further extend the findings among adults $[1,4,5]$ that neuroticism is a critical vulnerability factor in the initiation of depressive symptoms as early as adolescence. In contrast, all three personality traits and negative life events were associated with the occurrence of two episodes of depressive symptoms. Elevated neuroticism or psychoticism as well as more negative life events increased the risk of, whereas elevated extraversion protected against the recurrence of depressive symptoms. Because there were differential associations of personality traits with repeated episode versus single episode of depressive symptoms, our findings are less compatible with the common vulnerability hypothesis $[10,36]$. Instead, these imply that once an episode of depressive symptoms has been induced, it changes one's personality in such a way that future recurrent episode become more likely. In addition, sensitizations to both stressors and recurrent depressive symptoms might take place and the threshold of an episode of depressive symptoms was lowered such that minor exposure can lead to its recurrence. The lowering of threshold after the initiation of an episode of depressive symptoms is further supported by the doseresponse relationship between the increment in exposure and the recurrence of depressive symptoms.

Because the negative life events reported by this sample of nonreferred adolescents were mainly nonsevere in nature, we could not evaluate whether three or more severe life events was a critical threshold for the initiation of depressive symptoms, as postulated in the kindling model for depressive disorders [11-13]. Nevertheless, these less noxious but still troublesome life events appear to have cumulative effects on the recurrence of depressive symptoms in adolescents, consistent with studies in adults for depressive disorders $[11,14]$ and a study in adolescents [37] for depressive symptoms. However, the nonseverity of life events may also explain our failure to detect an interaction between life events and personality traits on the occurrence of depressive 
symptoms, which was demonstrated in a previous study of adult women [5].

The most frequently reported five life events by our subjects appear to reflect the current cultural environment in Taiwan. Middle school students today are still under tremendous pressure of advancing to a higher degree, most likely resulting from a traditional emphasis on diploma rather than talents. This may lead to adolescents' spending the majority of their time studying the course material, holding different opinions from those of their parents' on how to arrange their free time, and less quality time with their parents. Our findings that more negative life events were associated with the recurrence of depressive symptoms point to another possibility that the relationship between negative life events and early episodes of depressive symptoms might be reciprocal [38]. That is, the recurrent depressive symptoms could be precipitated by life stressors, which might be triggered by an earlier episode of symptoms [39]. Furthermore, the increasing vulnerability caused by the cumulative effect of life events may be a result of personality because people with elevated neuroticism or psychoticism are prone to make negative cognitive appraisals of situations requiring coping, which in turn, tends to make a stressful event much more stressful [40].

There are limitations of this study. First, our definition of an episode of depressive symptoms by means of a cutoff of CES-D score is not equivalent directly to a clinical diagnosis. The dichotomization of CES-D or dividing personality scale into three categorical levels might also lead to loss of information. Nevertheless, the findings of threshold-like features on the relations of personality and life events to episodes of depressive symptoms lend support to our choice of nondimensional approach. Second, the information on the change in life events over time, which was associated with chronic or repeated depression [39], was not available in this study. Thus, this study can only provide a preliminary view for the relationship between personality traits and life events on adolescents' short-term course of depressive symptoms. Third, the finding that more depressive symptoms and higher levels of neuroticism among students who were not followed up than those included in this study rendered the interpretation of our findings to be conservative. Last, because of the limited number of schools available (only six schools in Wave 1 and five schools in Wave 2), we did not conduct hierarchical linear modeling for the concern of biased standard errors for both individual and contextual variables.

These limitations notwithstanding, our results indicate that nonreferred adolescents are subject to similar risk factors for the initiation or recurrence of depressive symptoms as those found in adults or clinical populations. Our findings add to the growing literature documenting the close interrelationships between personality, negative life events, and depressive symptoms, and suggest that a comprehensive program addressing different domains of personality and negative life events is important for the prevention of adolescent depression.

\section{Acknowledgments}

This study was supported by grants from the National Science Council, Taipei (NSC89-2320-B-002-107 and NSC89-2314-B-002-473) and from the National Health Research Institute (PD9203). The authors would like to thank Dr. Jeff Wayman for his help in the multiple imputation analysis. We would also like to acknowledge the hard work of Ms. Y.-C. Chen in the fieldwork and thank the principals and teachers of the participating schools for their help in recruiting students.

\section{References}

[1] Katz R, McGuffin P. Neuroticism in familial depression. Psychol Med 1987; 17:155-61.

[2] Clark LA, Watson D, Mineka S. Temperament, personality, and the mood and anxiety disorders. J Abnorm Psychol 1994;103:103-16.

[3] Farmer A, Redman K, Harris T, et al. Neuroticism, extraversion, life events and depression. The Cardiff Depression Study. Br J Psychiatry 2002;181:118-22.

[4] Block JH, Gjerde PF, Block JH. Personality antecedents of depressive tendencies in 18-year-olds: a prospective study. J Pers Soc Psychol 1991;60:726-38.

[5] Kendler KS, Kuhn J, Prescott CA. The interrelationship of neuroticism, sex, and stressful life events in the prediction of episodes of major depression. Am J Psychiatry 2004;161:631-6.

[6] Berlanga C, Heinze G, Torres M, et al. Personality and clinical predictors of recurrence of depression. Psychiatr Serv 1999;50:37680 .

[7] Ormel J, Oldehinkel AJ, Vollebergh W. Vulnerability before, during, and after a major depressive episode. Arch Gen Psychiatry 2004;61: 990-6.

[8] Duggan C, Sham P, Lee A, et al. Neuroticism: a vulnerability marker for depression evidence from a family study. J Affect Disord 1995; 35:139-43.

[9] Kendler KS, Gatz M, Gardner CO, et al. Personality and major depression: a Swedish longitudinal, population-based twin study. Arch Gen Psychiatry 2006;63:1113-20.

[10] Burcusa SL, Iacono WG. Risk for recurrence in depression. Clin Psychol Rev 2007;doi:10.1016/j.cpr.2007.02.005.

[11] Franko DL, Striegel-Moore RH, Brown KM, et al. Expanding our understanding of the relationship between negative life events and depressive symptoms in black and white adolescent girls. Psychol Med 2004;34:1319-30.

[12] Bifulco A, Bernazzani O, Moran PM, et al. Lifetime stressors and recurrent depression: preliminary findings of the Adult Life Phase Interview (ALPHI). Soc Psychiatry Psychiatr Epidemiol 2000;35: 264-75.

[13] Post RM. Transduction of psychosocial stress into the neurobiology of recurrent affective disorder. Am J Psychiatry 1992;149:999-1010.

[14] Kendler KS, Thornton LM, Gardner CO. Stressful life events and previous episodes in the etiology of major depression in women: an evaluation of the "kindling" hypothesis. Am J Psychiatry 2000;157: 1243-51.

[15] Lewinsohn PM, Allen NB, Seeley JR, et al. First onset versus recurrence of depression: differential processes of psychosocial risk. J Abnorm Psychol 1999;108:483-9. 
[16] Kraaij V, Arensman E, Spinhoven P. Negative life events and depression in elderly persons: a meta-analysis. J Gerontol B Psychol Sci Soc Sci 2002;57:87-94.

[17] Monroe SM, Harkness KL. Life stress, the "kindling" hypothesis, and the recurrence of depression: considerations from a life stress perspective. Psychol Rev 2005;112:417-45.

[18] Ormel J, Oldehinkel AJ, Brilman EI. The interplay and etiological continuity of neuroticism, difficulties, and life events in the etiology of major and subsyndromal, first and recurrent depressive episodes in later life. Am J Psychiatry 2001;158:885-91.

[19] Zeiss AM, Lewinsohn PM. Enduring deficits after remissions of depression: a test of the scar hypothesis. Behav Res Ther 1988;26: $151-8$.

[20] McCleery JM, Goodwin GM. High and low neuroticism predict different cortisol responses to the combined dexamethasone- $\mathrm{CRH}$ test. Biol Psychiatry 2001;49:410-5.

[21] Uehara T, Sakado K, Sakado M, et al. Relationship between stress coping and personality in patients with major depressive disorder. Psychother Psychosom 1999;68:26-30.

[22] Bolger N, Zuckerman A. A framework for studying personality in the stress process. J Pers Soc Psychol 1995;69:890-902.

[23] Oldehinkel AJ, Wittchen H-U, Schuster P. Prevalence, 20-month incidence and outcome of unipolar depressive disorders in a community sample of adolescents. Psychol Med 1999;29:655-68.

[24] Yang HJ, Soong WT, Kuo P-H, et al. Using the CES-D in a twophase survey for depressive disorders among non-referred adolescents in Taiwan: an stratum-specific likelihood ratio analysis. J Affect Disord 2004;82:419-30.

[25] Harrington R, Fudge H, Rutter M, et al. Adult outcomes of childhood and adolescent depression. I. Psychiatric status. Arch Gen Psychiatry 1990;47:465-73.

[26] Ge X, Conger RD, Lorenz FO, et al. Multiple influences in parent and adolescent psychological distress. Dev Psychol 1995;31:406-19.

[27] Radloff LS. The CES-D scale: a self-report depression scale for research in general population. Appl Psychol Meas 1977;1:385-401.

[28] Jacobson NS, Truax P. Clinical significance: a statistical approach to defining meaningful change in psychotherapy research. J Consul Clin Psychol 1991;59:12-9.
[29] Geerling SW, Beekman ATF, Deeg DJH, et al. Physical health and the onset and persistence of depression in older adults: an eight-wave prospective community-based study. Psychol Med 2000;30:369-80.

[30] Eysenck HJ, Eysenck SBG. Manual of the Eysenck Personality Questionnaire. London: Hodder \& Stoughton Educational, 1975.

[31] Kuo PH, Chih YC, Soong WT, et al. Assessing personality features and their relations with behavioral problems in adolescents: Tridimensional Personality Questionnaire and Junior Eysenck Personality Questionnaire. Compr Psychiatry 2004;45:20-8.

[32] Johnson JH, McCutcheon SM. Assessing life stress in older children and adolescents: preliminary findings with the Life Events Checklist. In: Sarason IG, Spielberger CD, eds. Stress and Anxiety. Washington, DC: Hemisphere, 1980.

[33] Lewinsohn PM, Hops H, Roberts RE, et al. Adolescent psychopathology: I. Prevalence and incidence of depression and other DSMIII-R disorders in high school students. J Abnorm Psychol 1993;102: 133-44.

[34] Keller MB, Lavori PW, Mueller TI, et al. Time to recovery, chronicity, and levels of psychopathology in major depression. A 5-year prospective follow-up of 431 subjects. Arch Gen Psychiatry 1992;49: 809-816.

[35] Lewinsohn PM, Clarke GN, Seeley JR, et al. Major depression in community adolescents: age at onset, episode duration, and time to recurrence. J Am Acad Child Adolesc Psychiatry 1994;33:809-18.

[36] Krueger RF. The structure of common mental disorders. Arch Gen Psychiatry 1999;56:921-6.

[37] Lewinsohn PM, Hoberman HM, Rosenbaum M. A prospective study of risk factors for unipolar depression. J Abnorm Psychol 1988;97: 251-64.

[38] Patton GC, Coffey C, Posterino M, et al. Life events and early onset depression: cause or consequence? Psychol Med 2003;33:1203-10.

[39] Ge X, Conger RD, Elder GH Jr. Pubertal transition, stressful life events, and the emergence of gender differences in adolescent depressive symptoms. Dev Psychol 2001;37:404-17.

[40] Bolger N, Schilling EA. Personality and the problems of everyday life: the role of neuroticism in exposure and reactivity to daily stressors. J Pers 1991;59:355-86. 